\title{
Editorial
}

\section{Antidepressants: too little or too much?}

Ill health is common among elderly people as are depressive symptoms; they often coexist. Approximately one-third of elderly medical inpatients have depressive symptoms, often unrecognized and/or untreated. ${ }^{1}$ There are different possible explanations for this. Patients may be reluctant to discuss psychological problems or doctors too busy to listen: '. . . while I still wish to be communicative and caring the pressure of time and the need to protect myself have led me to ... get the blood, get the diagnosis, write the drugs up and disappear'. ${ }^{2}$ More importantly there may be doubts about the treatability of the 'depression' in this patient group and therefore a reluctance both to acknowledge and to search for it.

Screening questionnaires have been strongly advocated ${ }^{3}$ and they are good at eliciting depressive symptoms. However, depressive symptoms, even when severe, are of nonspecific diagnostic significance since they occur in a variety of both normal and pathological states. Physical illness affects people's mental well-being in ways which vary considerably. This may depend not only on the nature of the medical condition but on the particular individual affected, his or her personality attributes, and social circumstances. Not only can depression mimic symptoms of a medical illness but medical illness may produce symptoms that are identical to the neurovegetative symptoms of depression. Diagnostic accuracy is important to avoid the indiscriminate use of antidepressants.

A psychiatric assessment aims to confirm the diagnosis, evaluate suicide risk and exclude other significant psychiatric conditions. All available psychological, social and biological forms of treatment of depression may have applications in elderly medically ill patients but they are often overlooked. Many patients with depressive symptoms respond well to psychosocial interventions and support from the health care team; antidepressants are not a substitute for this. The ongoing doctor-patient relationship is often important to prevent despair and maintain hope for the future. Appropriate psychosocial interventions depend on understanding issues such as presence or absence of social supports, adjustments in family role(s), particular meaning and symbolic significance of the illness to the individual - including issues of body image and sexual function.

When the symptoms of depression are severe, persistent and unresponsive to appropriate medical and psychosocial interventions, consideration should be given to the addition of antidepressant medication. Prescribing for elderly people requires careful thought. ${ }^{4}$ Individual cases need to be assessed in terms of the relative risks and benefits but the potential for improving outcome with treatment is considerable.

Antidepressants are not a panacea for those with depression. Depressive symptoms in elderly patients need to be recognized, assessed and appropriate management instituted. All patients need social support and optimal medical care. It is important to remember that 'depression can be understandable and treatable'5; of those suffering from a major depressive illness, $60-70 \%$ will benefit from antidepressant treatment. It is not known for how long antidepressant medication should be continued and decisions should be made 


\section{Editorial}

on an individual basis, balancing potential adverse effects and benefits. Medication may need continuation in the long term for some patients to prevent relapse. 6 What is known is that depression among elderly people contributes to poor physical health, increased mortality and a poorer physical recovery. It also represents human suffering which is potentially remediable.

Andrew Thornton, Senior Registrar in Psychiatry of the Elderly and Robert Baldwin, Consultant Psychiatrist for the Elderly, York House, Manchester Royal Infirmary, Manchester $M 139 B X, U K$.

\section{References}

1 Koenig HG, Meador KG, Cohen HJ, Blazer DG

Detection and treatment of major depression in older medically ill hospitalised patients. Int J Psychiatry Med 1988; 18(1): 17-31.

2 D'Souza M. The longest day. BMJ 1994; 309: 1238.

3 Royal College of Physicians/British Geriatrics Society. Standardised assessment scales for elderly people. Report of joint workshops of the research unit of the Royal College of Physicians and the British Geriatrics Society. London: Royal College of Physicians, 1992.

4 Burns A, Baldwin R. Prescribing psychotropic drugs for the elderly. Adv Psychiatr Treatment 1994; 1: 23-31.

5 White PD. Clinical depression can be understandable and treatable. $B M J 1994 ; 309: 721$.

6 Old Age Depression Interest Group (OADIG). How long should the elderly take antidepressants? A double-blind placebo-controlled study of continuation/prophylaxis therapy. BrJ Psychiatry 1993; 162: 175-82. 